Schmerz 2016 $30: 294$

DOI 10.1007/s00482-016-0121-x

Online publiziert: 24. Mai 2016

(c) Deutsche Schmerzgesellschaft e.V. Published by Springer-Verlag Berlin Heidelberg - all rights reserved 2016

CrossMark

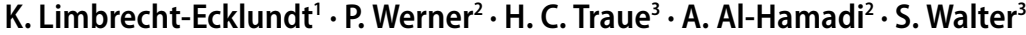

${ }^{1}$ Schmerzmedizin und Schmerzpsychologie, Klinik für Anaesthesiologie, Schmerzmedizin und Schmerzpsychologie, Zentrum für Anästhesiologie und Intensivmedizin, Klinik und Poliklinik für Anästhesiologie, Universitätsklinikum Hamburg-Eppendorf (UKE), Hamburg, Deutschland

${ }^{2}$ Institut für Informations- und Kommunikationstechnik, Otto-von-Guericke-Universität Magdeburg, Magdeburg, Deutschland

${ }^{3}$ Sektion Medizinische Psychologie, Universität UIm, UIm, Deutschland

\section{Erratum zu: Mimische Aktivität differenzierter Schmerzintensitäten. Korrelation der Merkmale von Facial Action Coding System und Elektromyographie}

Erratum zu:

Schmerz (2016)

DOI $10.1007 / \mathrm{s} 00482-016-0105-\mathrm{x}$

In der am 8. April 2016 veröffentlichten Online-Fassung dieses Beitrags ist bedauerlicherweise der Autor Ayoub AlHamadi nicht aufgeführt. Richtig ist:

K. Limbrecht-Ecklundt, P. Werner, H.C. Traue, A. Al-Hamadi, S. Walter.

In der Druckfassung ist dies korrekt angegeben.

\section{Korrespondenzadresse}

\section{Dr. K. Limbrecht-Ecklundt}

Schmerzmedizin und Schmerzpsychologie, Klinik für Anaesthesiologie, Schmerzmedizin und Schmerzpsychologie, Zentrum für Anästhesiologie und Intensivmedizin, Klinik und Poliklinik für Anästhesiologie, Universitätsklinikum Hamburg-Eppendorf (UKE)

Martinistraße 52, 20246 Hamburg, Deutschland k.limbrecht-ecklundt@uke.de
Die Online-Version des Originalartikels ist unter doi: 10.1007/s00482-016-0105-xzu finden. 